\title{
Evaluación de la confiabilidad del sistema de generación eléctrica utilizando elementos algebraicos
}

\author{
Reliability evaluation of the power generation system using algebraic \\ elements
}

Deysi Margoth Guanga Chunata, ${ }^{1}$ Luis Enrique Sarmiento Torres, ${ }^{2}$ Marianela de Jesús Inca Chunata. ${ }^{3}$

\section{Recibido: 15-05-2019 / Revisado: 18-06-2019 /Aceptado: 09-07-2019/ Publicado: 28-07-2019}

\begin{abstract}
.
DOI: https://doi.org/10.33262/cienciadigital.v3i3.2.1.818

This article presents the application of a Bayesian model that consists in the implementation of an infinite state Markov chain for the evaluation of the reliability of the electric generation system of three generators. The analysis will allow obtaining a model of the current and future operating behavior of three generator sets. The data to analyze are the operative registers elaborated by the operators of the generators, who in their logbook write down the operation and failure times. The analysis sample comprises five (05) years, in twelve-hour intervals. Therefore, with the development of the present model it will be possible to predict parameters and indicators such as: availability, maintainability, reliability, failure rate, repair rates, average time for repair and average time between failures; with the purpose of achieving a better management of the assets within the maintenance management, having as a case of application that of the electric generation system.
\end{abstract}

Keywords: Reliability, Active Indicator, Markov, Failure, Vector.

\section{Resumen.}

Este artículo presenta la aplicación de un modelo Bayesiano que consiste en la implementación de cadena de Markov de estado finito para la evaluación de la confiabilidad del sistema de generación eléctrica de tres grupos electrógenos. El análisis permitirá obtener un modelo del comportamiento de operación actual y futuro

\footnotetext{
${ }^{1}$ Escuela Superior Politécnica de Chimborazo, Chimborazo Ecuador. deysiguanga@espoch.edu.ec

${ }^{2}$ Petroamazonas EP, Sucumbíos, Ecuador. luis_sarmiento@petroamazonas.ec

${ }^{3}$ Secretaría Nacional de Educación Superior, Pichincha, Ecuador. minca@senescyt.gob.ec
} 
de tres grupos electrógenos. Los datos a analizar son los registros operativos elaborados por los operadores de los grupos electrógenos, quienes en su bitácora anotan los tiempos de operación y falla. La muestra de análisis comprende cinco (05) años, en intervalos de doce horas. Por lo tanto, con el desarrollo de la presente modelación se logrará predecir parámetros e indicadores relacionados a la confiabilidad, tasa de fallas, tasas de reparación, tiempo medio para reparar y tiempo medio entre fallas; con la finalidad de conseguir una mejor gestión de los activos dentro de la gestión de mantenimiento, teniendo como caso de aplicación el del sistema de generación eléctrica.

Palabras claves: Confiabilidad, Indicador Activo, Markov, Falla, Vector.

\section{Introducción.}

Los conceptos estadísticos, necesarios para la aplicación del análisis de confiabilidad son herramientas de análisis que comprenden distribuciones, modelos e indicadores necesarios para interpretar el comportamiento de un activo. La suposición de que la detección de fallos de un grupo electrógeno se puede modelar a través de una cadena de Markov aplicado al diseño de varios tipos de simuladores de baja complejidad, que luego deben procesarse para determinar si se comete un error de decisión cuyo resultado es la inoperatividad de los equipos.

Por más de medio siglo, los investigadores han tratado de adaptarse al tiempo discreto de medición de fallos del sistema basado en modelos que van desde los primeros canales GilbertElliott de dos estados a más complejos modelos como los basados en cadenas ocultas de Markov (Garcia, P. 1997).. El Canal de Markov del Estado Finito (FSMC) posee buen equilibrio entre la precisión y la complejidad (Wang, H. 1997).. El modelamiento se basa en la estructura de un número finito de estados logrando que el uso de un proceso de Markov de primer orden sea una buena aproximación usando la teoría de la información (Tan, C. 2000)..

Los problemas de Markov vistos como una matriz asociada al comportamiento lineal de los grupos electrógenos conducen a problemas de valores propios si se desea conocer el estado límite del proceso en el cual el vector del estado X se reproduce bajo la multiplicación por la matriz estocástica A que rige el proceso.

\section{Metodología.}

Para el desarrollo del modelamiento fue necesaria la recopilación de fallos en cada parte del sistema generador discretizados en el tiempo con un registro histórico de cinco años. El análisis de los datos se lo realizó mediante el programa estadístico SPSS. La asociación de variables para la construcción de la matriz estocástica se la desarrolló empleando las medias de los fallos contemplados por cada generador. A continuación, se detalla los principales pasos seguidos para obtener los resultados: 


\section{Deducción del modelo matemático}

Se seleccionó dos (03) grupos electrógenos para la implementación de indicadores y registro de fallas, a quienes se los conocerá como MG1, MG2 y MG3 en adelante, como se muestra en la Tabla 1. La secuencia de fallas y de reparaciones de un componente reparable define un proceso aleatorio que se puede representar como una cadena de Markov de dos estados discretos y tiempo continuo cuya representación se muestra a continuación:

Figura 1. Modelo reparable de dos estados

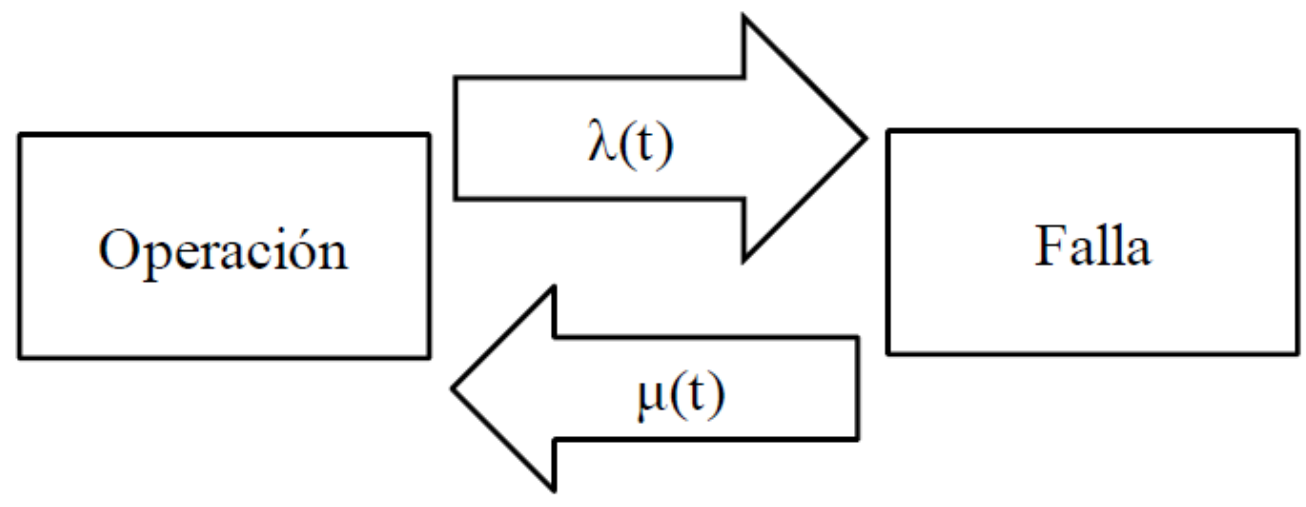

Fuente: Elaboración propia

Las tasas de transición entre estados se definen:

\begin{tabular}{|c|c|}
\hline Tasa de fallas & $\lambda(t)=1 / E\left(T_{f}\right)=\frac{d E\left[n_{f}(t)\right]}{d t}$ \\
\hline Tasa de reparaciones & $\mu(t)=1 / E\left(T_{r}\right)=\frac{d E\left[n_{r}(t)\right]}{d t}$ \\
\hline
\end{tabular}

Para un sistema de " $n$ " componentes, cada uno con dos estados operativos, el número total de estados operativos del sistema viene dado por $2^{n}$.

Si se considerase un intervalo de tiempo $d t$, muy pequeño, de tal manera que la probabilidad de que ocurran más de una falla o más de una reparación sea muy pequeña y por lo tanto pueda despreciarse la ocurrencia de estos eventos (Wang, H. 1997).. Así:

Probabilidad de una falla en $\mathrm{t}=$ Probabilidad de una falla en $(t+d t)=\lambda(t) d t$. 
Probabilidad de una reparación en $\mathrm{t}=$ Probabilidad de una reparación en $(t+d t)=\mu(t) d t$.

La probabilidad de estar en el estado operativo después de un intervalo de tiempo $d t$ es igual a la probabilidad de estar operativo en $\mathrm{t}$ y no haber fallado en $d t+$ probabilidad de estar fallado en $t$ y haber sido reparado en $d t$ :

$P 1(t+d t)=P 1(t)[1-\lambda(t) d t]+P 2(t)[\mu(t) d t]$

La probabilidad de estar en el estado de reparación (fallado) después de un intervalo de tiempo $d t$ es igual a la probabilidad de estar fallado en $t$ y no haber sido reparado en $d t+$ probabilidad de estar no fallado en $\mathrm{t}$ y haber fallado en $d t$ :

$P 2(t+d t)=P 2(t)[1-\mu(t) d t]+P 1(t)[\lambda(t) d t]$

Así reorganizando términos en ambas ecuaciones:

De (2.1):

$P 1(t+d t)=P 1(t)[1-\lambda(t) d t]+P 2(t)[\mu(t) d t]$

$P 1(t+d t)=P 1(t)-\lambda(t) d t P 1(t)+\mu(t) d t P 2(t)$

$P 1(t+d t)-P 1(t)=[-\lambda(t) P 1(t)+\mu(t) P 2(t)] d t$

$P 1(t+d t)-P 1(t) / d t=-\lambda(t) P 1(t)+\mu(t) P 2(t)$

$\frac{\mathrm{P} 1(\mathrm{t}+\mathrm{dt})-\mathrm{P} 1(\mathrm{t})}{d t}=-\lambda(t) P 1(t)+\mu(t) P 2(t)$

Se obtiene:

$\frac{\mathrm{dP} 1(\mathrm{t})}{d t}==-\lambda(\mathrm{t}) \mathrm{P} 1(\mathrm{t})+\mu(\mathrm{t}) \mathrm{P} 2(\mathrm{t})$

De (2.2):

$P 2(t+d t)=P 2(t)[1-\mu(t) d t]+P 1(t)[\lambda(t) d t]$

$P 2(t+d t)=P 2(t)-\mu(t) d t P 2(t)+\lambda(t) d t P 1(t)$

$P 2(t+d t)-P 2(t)=[\lambda(t) P 1(t)-\mu(t) P 2(t)] d t$ 
Lo cual resulta en el siguiente sistema de ecuaciones diferenciales ordinarias:

$$
\begin{aligned}
& \frac{\mathrm{dP} 1(\mathrm{t})}{\mathrm{dt}}=-\lambda(\mathrm{t}) \mathrm{P} 1(\mathrm{t})+\mu(\mathrm{t}) \mathrm{P} 2(\mathrm{t}) \\
& \frac{\mathrm{dP} 2(\mathrm{t})}{\mathrm{dt}}=\lambda(\mathrm{t}) \mathrm{P} 1(\mathrm{t})-\mu(\mathrm{t}) \mathrm{P} 2(\mathrm{t})
\end{aligned}
$$

El cual se puede expresar en alguna de las siguientes formas:

$$
\begin{aligned}
& \frac{\frac{\mathrm{dP} 1(\mathrm{t})}{d t}}{\frac{\mathrm{dP} 2(\mathrm{t})}{d t}}=\left(\begin{array}{cc}
-\lambda(\mathrm{t}) & \mu(\mathrm{t}) \\
\lambda(\mathrm{t}) & -\mu(\mathrm{t})
\end{array}\right)\left(\begin{array}{l}
\mathrm{P} 1(\mathrm{t}) \\
\mathrm{P} 2(\mathrm{t})
\end{array}\right) \\
& \frac{p_{1}(t)}{p_{2}(t)}=\left(\begin{array}{cc}
-\lambda(\mathrm{t}) & \mu(\mathrm{t}) \\
\lambda(\mathrm{t}) & -\mu(\mathrm{t})
\end{array}\right)\left(\begin{array}{l}
\mathrm{P} 1(\mathrm{t}) \\
\mathrm{P} 2(\mathrm{t})
\end{array}\right) \\
& \overline{P(t)}=H^{T} \overline{P(t)}
\end{aligned}
$$

Donde $\mathrm{H}$ es la matriz estocástica de tasas de transición entre estados.

La solución de la cadena de Markov son las probabilidades de ocurrencia de los estados 1 y 2 como una función del tiempo (Wang, H. 1997).:

\begin{tabular}{|l|l|l|}
\hline$P 1(t)$ & $\begin{array}{l}\text { Es la disponibilidad } \\
\text { instantánea } \\
\text { componente. }\end{array}$ & $\begin{array}{l}\text { Es la probabilidad de encontrar el componente en el } \\
\text { estado "bueno" en algún instante del tiempo }\end{array}$ \\
\hline $\begin{array}{l}P(t) \\
=U(t)\end{array}$ & $\begin{array}{l}\text { Es la indisponibilidad } \\
\text { instantánea del componente }\end{array}$ & $\begin{array}{l}\text { Es la probabilidad de encontrar el componente en el } \\
\text { estado "fallado" en algún instante del tiempo }\end{array}$ \\
\hline
\end{tabular}

Si los procesos de fallas y de reparaciones del componente reparable son estacionarios, independientes y ajustan a distribuciones de probabilidad, se podrá entonces utilizar como modelo de confiabilidad una cadena de Markov homogénea (García, P. 1997).. 
Como la tasa de falla y de reparación son constantes, éstas se pueden escribir como:

\begin{tabular}{l|l}
\hline$\lambda(t)=\lambda=1 / E(T f)$ & $\mu(t)=\mu=1 / E(T r)$
\end{tabular}

La cadena de Markov homogénea se define entonces por:

\begin{tabular}{|c|c|c|}
\hline Ftf $(t)$ & \begin{tabular}{|lr} 
La función & de \\
distribución de & des \\
probabilidad de & los \\
tiempos para falla. &
\end{tabular} & \begin{tabular}{|lcccr} 
También se & puede & utilizar & la \\
correspondiente & & función & de \\
densidad & de & probabilidad \\
$f t f(t)$. & & & &
\end{tabular} \\
\hline$F \operatorname{Ftr}(t)$ & $\begin{array}{lrr}\text { La función } & \text { de } \\
\text { distribución } & \text { de } \\
\text { probabilidad } & \text { de } & \text { los } \\
\text { tiempos } & & \text { para } \\
\text { reparación. } & & \end{array}$ & 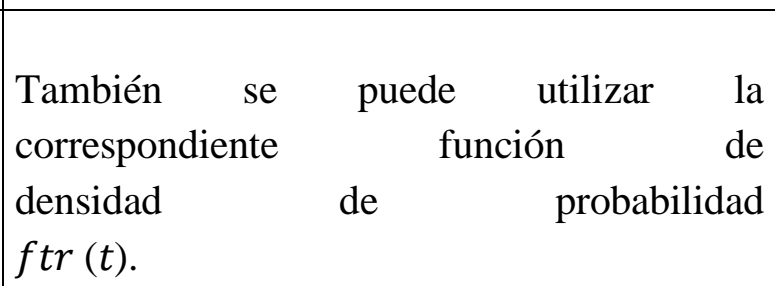 \\
\hline
\end{tabular}

\section{SOLUCIÓN ESTADÍSTICA DE LA CADENA DE MARKOV}

Si se tiene evidencia de que las muestras de tiempos para fallas y de tiempos para reparación son estacionarias, también es posible calcular en forma estadística las disponibilidades e indisponibilidades de estado estable y las tasas de eventos del modelo de confiabilidad del componente reparable de dos estados, de la siguiente forma (Wang, H. 1997).:

$$
\begin{array}{ll}
P_{1}(\infty)=A=\frac{\sum t_{f}}{T} & P_{2}(\infty)=A=\frac{\sum t_{r}}{T} \\
\lambda=\frac{n_{f}}{T-\sum t_{r}}=\frac{n_{f}}{\sum t_{r}} & \mu=\frac{n_{r}}{T-\sum t_{f}}=\frac{r}{\sum t_{f}}
\end{array}
$$

\section{RESULTADOS}

Es preciso saber que la calidad de estos cálculos depende del tamaño de la muestra. Con los datos analizados de las tasas de falla $(\lambda)$ y reparación $(\mu)$ de los grupos electrógenos, se obtiene la matriz de transición y estados, según Markov: 
Tabla 1 Datos iniciales de operación y falla

\begin{tabular}{lllll}
\hline UNIDAD & $\begin{array}{l}\text { CAPACIDAD } \\
(\mathrm{KW})\end{array}$ & $\begin{array}{l}\text { FOR } \\
(\%)\end{array}$ & $\begin{array}{l}\lambda \\
\text { (fallas/hora) }\end{array}$ & $\begin{array}{l}\mu \\
\text { (reparación/horas) }\end{array}$ \\
\hline GEN 1 & 850 & 0.58 & 0.00057 & 0.19 \\
GEN 2 & 1175 & 9.05 & 0.00251 & 0.0892 \\
GEN 3 & 1250 & 14.47 & 0.0024 & 0.0908 \\
\hline
\end{tabular}

Fuente: Elaboración propia

FOR: Tasa de salidas forzadas (Forced Outage Rate)

El sistema tiene $2^{3}=8$ estados descritos así

(A: disponible; $\mathrm{U}$ : indisponible)

El modelo de Markov da probabilidades de los estados posibles en un sistema en función del tiempo.

Tabla 2 Estados de disponibilidad e indisponibilidad de los grupos electrógenos

\begin{tabular}{llllll}
\hline & Estado & & & & \\
\hline & GEN1 & GEN2 & GEN3 & $\begin{array}{l}\text { KW } \\
\text { Disponibles }\end{array}$ & $\begin{array}{l}\text { KW } \\
\text { perdidos }\end{array}$ \\
1 & U & U & U & 0 & 3275 \\
2 & A & U & U & 850 & 2425 \\
3 & U & A & U & 1175 & 2100 \\
4 & U & U & A & 1250 & 2025 \\
5 & A & A & U & 2025 & 1250 \\
6 & A & U & A & 2100 & 1175 \\
7 & U & A & A & 2425 & 850 \\
8 & A & A & A & 3275 & 0 \\
\hline
\end{tabular}

Fuente: Elaboración propia 
Las tasas de transición entre estados (hij) forman la matriz h:

\begin{tabular}{|c|c|c|c|c|c|c|c|}
\hline $\begin{array}{c}-\mu 1 \\
-\mu 2-\mu 3\end{array}$ & $\mu 1$ & $\mu 2$ & $\mu 3$ & 0 & 0 & 0 & 0 \\
\hline$\lambda 1$ & $\begin{array}{c}-\lambda 1 \\
-\mu 2-\mu 3\end{array}$ & 0 & 0 & $\mu 2$ & $\mu 3$ & 0 & 0 \\
\hline$\lambda 2$ & 0 & $\begin{array}{c}-\mu 1 \\
-\lambda 2-\mu 3\end{array}$ & 0 & $\mu 1$ & 0 & $\mu 3$ & 0 \\
\hline$\lambda 3$ & 0 & 0 & $\begin{array}{c}-\mu 1 \\
-\mu 2-\lambda 3\end{array}$ & 0 & $\mu 1$ & $\mu 2$ & 0 \\
\hline 0 & $\lambda 2$ & $\lambda 1$ & 0 & $-\lambda 1$ & 0 & 0 & $\mu 3$ \\
\hline 0 & $\lambda 3$ & 0 & $\lambda 1$ & 0 & $\begin{array}{c}-\lambda 1 \\
-\mu 2-\lambda 3\end{array}$ & 0 & $\mu 2$ \\
\hline 0 & 0 & $\lambda 3$ & $\lambda 2$ & 0 & 0 & $\begin{array}{c}-\mu 1 \\
-\lambda 2-\lambda 3\end{array}$ & $\mu 1$ \\
\hline 0 & 0 & 0 & 0 & $\lambda 3$ & $\lambda 2$ & $\lambda 1$ & $\begin{array}{c}-\lambda 1 \\
-\lambda 2-\lambda 3\end{array}$ \\
\hline
\end{tabular}

Cada celda de la matriz contiene la probabilidad de transición Pij del estado i al estado j.

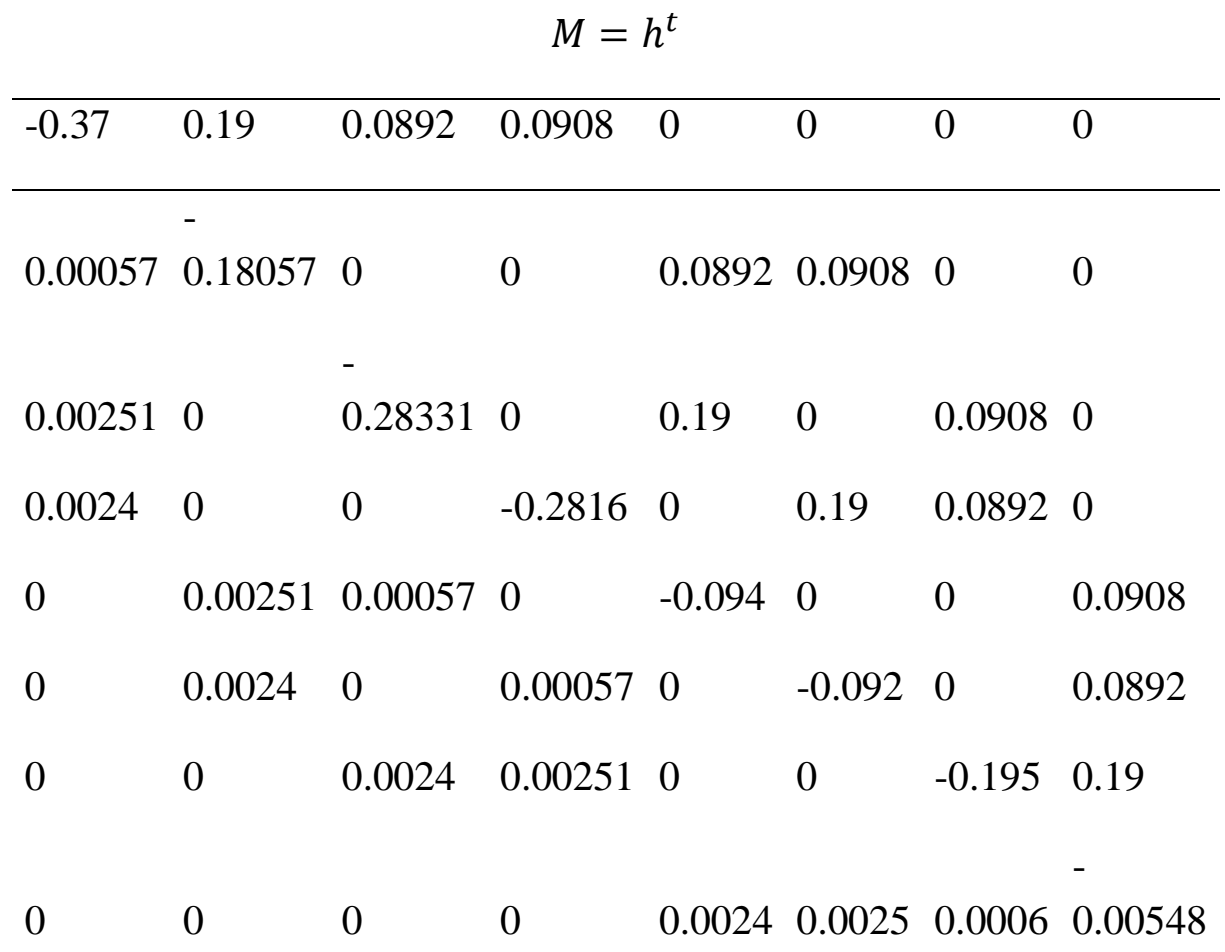


En estos sistemas, componentes o elementos poseen en general salidas que afectan su disponibilidad (Wang, H. 1997).. Éstas pueden ser:

Tabla 3 Tipos de salidas en un sistema reparable

\begin{tabular}{|c|c|c|c|}
\hline Tipo de salida & Descripción & Ejemplo & \\
\hline No planeadas & \begin{tabular}{llr} 
Son & de & \multicolumn{2}{c}{ naturaleza } \\
aleatoria en cuanto a su \\
ocurrencia y duración.
\end{tabular} & $\begin{array}{l}\text { Fallas } \\
\text { componente } \\
\text { vandalismo, } \\
\text { inundaciones, etc. }\end{array}$ & $\begin{array}{r}\text { de } \\
\text { sistema, } \\
\text { incendios, }\end{array}$ \\
\hline Planeadas & $\begin{array}{lr}\text { Se conoce de antemano } \\
\text { cuándo ocurrirán. } \\
\text { pueden pesponer. } \\
\text { determinísticas } \\
\text { cuanto a su ocurrencia } \\
\text { pero aleatorias } \\
\text { cuanto a su duración. }\end{array}$ & $\begin{array}{l}\text { Mantenimiento } \\
\text { preventivo, ir } \\
\text { toma de } \\
\text { ampliación } \\
\text { mejoramiento } \\
\text { sistema, solicitu } \\
\text { entidad externa. }\end{array}$ & $\begin{array}{r}\text { inspección, } \\
\text { medidas, } \\
\text { o del } \\
\text { de }\end{array}$ \\
\hline
\end{tabular}

Fuente: Elaboración propia

Las salidas planeadas pueden tratarse de la siguiente manera:

1. Determinar la programación de salidas planeadas del componente: en este caso al estudiar o generar la secuencia operativa del componente se debe mirar en qué períodos de tiempo estará indisponible según el programa de mantenimiento preventivo.

2. Incorporarlas al modelo probabilístico: implica asumir que se conoce que el componente tendrá salidas planeadas, pero no se conoce su programación. Válido para estudios de largo plazo o para componentes donde el mantenimiento preventivo no obedece a un programa riguroso.

Otro asunto, es que las salidas no planeadas y las salidas planeadas deben incorporarse en los índices de confiabilidad pues para algunos componentes del sistema eléctrico éstas salidas pueden tener una participación muy alta

\section{CONCLUSIONES}

Con base en los registros operativos de un sistema se puede considerar las siguientes conclusiones: 
- Se pueden aproximar los índices de confiabilidad que son estadísticas descriptivas, por ejemplo, el tiempo promedio para falla, las horas de indisponibilidad por año, etc.

- Con los índices de confiabilidad se pueden hacer los siguientes análisis:

$\checkmark$ Evaluar el desempeño del componente con respecto a valores de referencia u objetivo.

$\checkmark$ Plantear medidas correctivas para mejorar la confiabilidad del componente.

- Con los modelos probabilísticos se pueden hacer los siguientes análisis:

$\checkmark$ Simular la operación pasada y presente del componente.

$\checkmark$ Predecir el comportamiento futuro del componente.

- Para una mejor cognición se deben tratar los vectores como un elemento concreto y representable visualmente, como en el plano usando flechas desde el origen, considerando que a medida que el estudiante aprende más sobre álgebra lineal, es conveniente tener en cuenta que estos conocimientos son aplicables a un nivel mucho más general y que éste es el motivo por el cual los textos estudian el tema de un modo más abstracto

- El presente trabajo constituye una herramienta para el aprendizaje cognitivo de los espacios vectoriales abstractos. El resultado de la estrategia didáctica cognitiva permite a los alumnos su construcción al menos como proceso de aprendizaje dejando de lado la memorización.

\section{Referencias Bibliográficas.}

M. Mushkin and I. Bar-David, "Capacity and coding for the Gilbert-Elliott channels," IEEE Trans. on Info. Theory, vol. 35, pp. 1277-1290, Nov. 1989.

J. Garcia-Frias and P. M. Crespo, "Hidden Markov models for burst error characterization in indoor radio channels," IEEE Trans. on Veh. Tech., vol. 46, no. 4, pp. 1006-1020, Nov. 1997.

H.S. Wang and N. Moayeri, "Finite-state Markov channel- a useful model for radio communication channels," IEEE Trans. on Veh. Tech., vol. 44, no. 1, pp. 163-171, Feb. 1995.

H.S. Wang and P.-C. Chang, "On verifying the first-order Markovian assumption for a Rayleigh fading channel," IEEE Trans. on Veh. Tech., vol. 45, no. 2, pp. 353-357, May 1996. 
C. C. Tan and N. C. Beaulieu, "On first-order Markov modeling for the Rayleigh fading channel," IEEE Trans. on Comm., vol. 48, no. 12, pp. 2032-2040, Dec. 2000.

Troffé, M. (2009). PETROTECNIA Revista del Instituto Argentino del Petróleo y el Gas. $\begin{array}{llllll}\text { Recuperado el } & 24 & \text { de } & \text { Marzo }\end{array}$ http://www.petrotecnia.com.ar/febrero09/base_de_datos_de_confiabilidad.pdf

ISO 14224. (2006). Petroleum and natual gas industries - Collection and exchange of reliability and Maintenance data equipment. Switzerland: ISO.

Rockwell Automation. (2004). Recommendations For Writing VisualBasic for Applications (VBA) Code In RSViewSE - V1.3.

IEC 60812:2006 (E). (2006). Analysis techniques for system reliability - Procedure for failure mode and effects analysis (FMEA).

Petroamazonas EP - AMS. (2013). Análisis de Mantenimiento Centrado en Confiabilidad (RCM) de La Planta de Generación Eléctrica, Palo Azul, B18, PAM-EP. Petroamazonas EP, Mantenimiento, Quito.

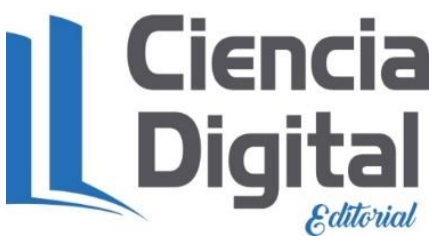




\section{PARA CITAR EL ARTÍCULO INDEXADO.}

Guanga Chunata, D., Sarmiento Torres, L., \& Inca Chunata, M. de J. (2019). Evaluación de la confiabilidad del sistema de generación eléctrica utilizando elementos algebraicos. Ciencia Digital, 3(3.2.1), 275-286. https://doi.org/10.33262/cienciadigital.v3i3.2.1.818

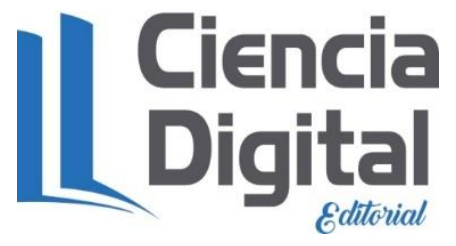

El artículo que se publica es de exclusiva responsabilidad de los autores y no necesariamente reflejan el pensamiento de la Revista Ciencia Digital.

El artículo queda en propiedad de la revista y, por tanto, su publicación parcial y/o total en otro medio tiene que ser autorizado por el director de la Revista Ciencia Digital.
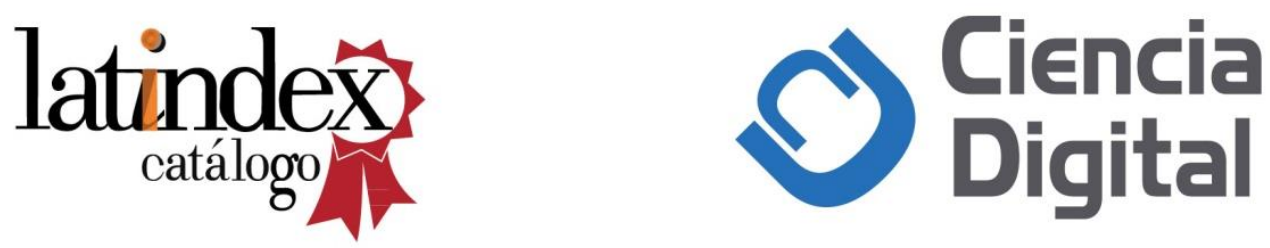